\title{
Front Matter: Volume 10912
}

, "Front Matter: Volume 10912," Proc. SPIE 10912, Physics and Simulation of Optoelectronic Devices XXVII, 1091201 (7 May 2019); doi:

10.1117/12.2531049

SPIE. Event: SPIE OPTO, 2019, San Francisco, California, United States 


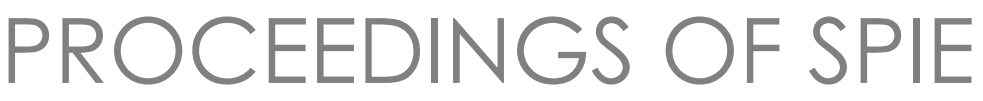

\title{
Physics and Simulation of Optoelectronic Devices XXVII
}

\author{
Bernd Witzigmann \\ Marek Osiński \\ Yasuhiko Arakawa \\ Editors
}

5-7 February 2019

San Francisco, California, United States

Sponsored and Published by

SPIE 
The papers in this volume were part of the technical conference cited on the cover and title page. Papers were selected and subject to review by the editors and conference program committee. Some conference presentations may not be available for publication. Additional papers and presentation recordings may be available online in the SPIE Digital Library at SPIEDigitalLibrary.org.

The papers reflect the work and thoughts of the authors and are published herein as submitted. The publisher is not responsible for the validity of the information or for any outcomes resulting from reliance thereon.

Please use the following format to cite material from these proceedings:

Author(s), "Title of Paper," in Physics and Simulation of Optoelectronic Devices XXVII, edited by Bernd Witzigmann, Marek Osiński, Yasuhiko Arakawa, Proceedings of SPIE Vol. 10912 (SPIE, Bellingham, WA, 2019) Seven-digit Article CID Number.

ISSN: 0277-786X

ISSN: 1996-756X (electronic)

ISBN: 9781510624665

ISBN: 9781510624672 (electronic)

Published by

SPIE

P.O. Box 10, Bellingham, Washington 98227-0010 USA

Telephone +1 3606763290 (Pacific Time) · Fax +1 3606471445

SPIE.org

Copyright (C) 2019, Society of Photo-Optical Instrumentation Engineers.

Copying of material in this book for internal or personal use, or for the internal or personal use of specific clients, beyond the fair use provisions granted by the U.S. Copyright Law is authorized by SPIE subject to payment of copying fees. The Transactional Reporting Service base fee for this volume is $\$ 18.00$ per article (or portion thereof), which should be paid directly to the Copyright Clearance Center (CCC), 222 Rosewood Drive, Danvers, MA 01923. Payment may also be made electronically through CCC Online at copyright.com. Other copying for republication, resale, advertising or promotion, or any form of systematic or multiple reproduction of any material in this book is prohibited except with permission in writing from the publisher. The CCC fee code is 0277$786 \mathrm{X} / 19 / \$ 18.00$.

Printed in the United States of America by Curran Associates, Inc., under license from SPIE.

Publication of record for individual papers is online in the SPIE Digital Library.

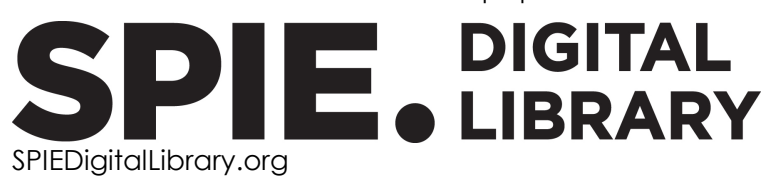

Paper Numbering: Proceedings of SPIE follow an e-First publication model. A unique citation identifier (CID) number is assigned to each article at the time of publication. Utilization of CIDs allows articles to be fully citable as soon as they are published online, and connects the same identifier to all online and print versions of the publication. SPIE uses a seven-digit CID article numbering system structured as follows:

- The first five digits correspond to the SPIE volume number.

- The last two digits indicate publication order within the volume using a Base 36 numbering system employing both numerals and letters. These two-number sets start with 00, 01, 02, 03, 04, 05, 06, 07, 08, 09, OA, OB ... 0Z, followed by 10-1Z, 20-2Z, etc. The CID Number appears on each page of the manuscript. 


\title{
Contents
}

\author{
vii Authors \\ ix Conference Committee
}

NANO-EMITTERS AND NON-CLASSICAL LIGHT

$1091205 \quad$ Photonic engineering providing conditions for direct exciton macroscopic coherence at elevated temperatures [10912-4]

III-NITRIDE LASERS AND LEDS

10912 OD Inhomogeneous spectral broadening in deep ultraviolet light emitting diodes [10912-12]

10912 OE InGaN/GaN $\mu$ LED SPICE modelling with size-dependent ABC model integration [10912-13]

\section{LASERS AND LEDs I}

10912 OG VENUS: a comprehensive electro-thermo-opto VCSEL simulator (Invited Paper) [10912-15]

$10912 \mathrm{OH} \quad$ Low-noise $\mathrm{x}$-band tunable microwave generator based on external cavity lasers [10912-16]

10912 Ol Characterization of MBE-grown 1.55- $\quad$ m GaSb-based multiple-quantum-well lasers at cryogenic temperatures [10912-17]

\section{LASER DYNAMICS}

$109120 \mathrm{M}$ Stable and unstable Nozaki-Bekki holes in a long laser [10912-21]

$10912 \mathrm{ON}$ Theoretical investigation on effects of group-velocity dispersion on mid-infrared quantumcascade lasers with Fabry-Perot and ring cavities [10912-22]

1091200 Coexisting periodic regimes in semiconductor lasers with optical feedback [10912-23] 


\section{ELECTROMAGNETICS AND PLASMONICS}

$109120 Q \quad$ Revisiting beamforming under the prism of inverse methods [10912-25]

10912 OT Ellipso-polarimetric schemes for improved surface plasmon resonance detection [10912-28]

\section{LASERS AND LEDs II}

10912 OU Hybrid modeling of quantum light emitting diodes: self-consistent coupling of drift-diffusion, Schrödinger-Poisson, and quantum master equations (Invited Paper) [10912-29]

10912 OV Carrier lifetime of heavily $\mathbf{p}$-doped base in light-emitting transistors and transistor lasers [10912-30]

$109120 \mathrm{X}$ Wide-temperature-range characterization of 1.55- $\mu \mathrm{m}$ phosphorus-free multiple-quantum-well lasers grown by MBE on InP [10912-32]

\section{PHOTODETECTION}

10912 OY Minimization of dark counts in PureB SPADs for NUV/VUV/EUV light detection by employing a 2D TCAD-based simulation environment [10912-33]

$109120 Z$ A comparative study of methods and algorithms for spatially resolved spectral value reconstruction with multispectral resolving filter-on-chip CMOS sensors [10912-34]

\section{SENSORS}

1091213 Bimetallic surface plasmon resonance photonic crystal fiber biosensor using refractory plasmonic material [10912-40]

1091214 Design and analysis of low-cost high-sensitive surface plasmon resonance-based biosensor [10912-41]

\section{OPTICAL SYSTEMS SIMULATION}

$1091215 \quad$ Fully tensorial elastic-wave mode solver for stimulated Brillouin scattering simulations in integrated photonics [10912-42]

$1091216 \quad$ Exploring the impacts of long-period corrugation and phase gratings on a cascade of phaseshifted lithium niobate waveguides with the combined theoretical and experimental approaches [10912-43]

1091217 Indoor positioning and intuitive advertising using visible light communication [10912-44] 
1091218 The design of universal logic gates using microring resonator structures [10912-45]

POSTER SESSION

1091219 Signal and crosstalk analysis using optical convolution of transmitted optical signals [10912-46]

$109121 \mathrm{~A} \quad$ Optical simulation for illumination using GPGPU ray tracing [10912-47]

10912 1B Design and analysis of surface-plasmon resonance (SPR) sensor to measure electric field using angle interrogation [10912-48]

10912 1C Circularly polarized lasing of ultraviolet plasmonic gammadion nanocavity [10912-49]

10912 1D Thermal properties and extinction of a wire-grid polarizer [10912-50]

10912 IF Metallo-dielectric Yagi-Uda nanoantennas based on rectangular shaped elements [10912-52]

$109121 \mathrm{G} \quad \mathrm{X}$-ray luminescence in undoped and bismuth-doped single crystal hybrid lead halide perovskites [10912-53]

$109121 \mathrm{H} \quad$ Multi-functional plasmonic biosensor based on alcohol-filled PCF [10912-54]

$1091211 \quad$ Design of photonic crystal fiber for efficient supercontinuum generation [10912-55]

$109121 \mathrm{~J} \quad$ Compact polarization splitter based on plasmonic micro-structured fiber [10912-56]

$109121 \mathrm{~K} \quad$ Optical design of dual mode seeker for short-wave infrared and four quadrant detectors in missile application [10912-57]

$109121 \mathrm{~L} \quad$ Compact seeker design by using piezo actuator in missile application [10912-58]

$109121 \mathrm{M}$ Saturation effects in nonlinear loop mirror lasers: square wave operation [10912-59]

$109121 \mathrm{~N}$ Designing artificial neural networks for band structures computations in photonic crystals [10912-60]

$1091210 \quad$ Fabrication and simulation of silver nanostructures on different types of porous silicon for surface enhanced Raman spectroscopy [10912-61]

$109121 Q \quad$ Optical propagation through layered anisotropic materials using effective medium theory [10912-63] 
Proc. of SPIE Vol. 10912 1091201-6

Downloaded From: https://www.spiedigitallibrary.org/conference-proceedings-of-spie on 25 Apr 2023 Terms of Use: https://www.spiedigitallibrary.org/terms-of-use 


\section{Authors}

Numbers in the index correspond to the last two digits of the seven-digit citation identifier (CID) article numbering system used in Proceedings of SPIE. The first five digits reflect the volume number. Base 36 numbering is employed for the last two digits and indicates the order of articles within the volume. Numbers start with 00, 01, 02, 03, 04, 05, 06, 07, 08, 09, OA, OB...0Z, followed by 10-1Z, 20-2Z, etc.

\author{
Abdulhalim, Ibrahim, OT \\ Addamane, Sadhvikas J., 0I, OX \\ Al-Ghezi, Hammid, 1Q \\ Azab, Mohammad Y., 13, 1H \\ Azhigulov, Dias, 18 \\ Bai, Jing, ON \\ Balakrishnan, Ganesh, Ol, OX \\ Bandarenka, Hanna, 10 \\ Banerjee, Partha P., 1Q \\ Bayram, Alican, 1K, 1L \\ Bertazzi, Francesco, OG \\ Birowosuto, Muhammad Danang, IG \\ Buch, B., $\mathrm{OZ}$ \\ Chang, Chiao-Yun, 1C \\ Chang, Shu-Wei, OV, IC \\ Chembo, Yanne K., $\mathrm{OH}$ \\ Cheng, Pi-Ju, OV, 1C \\ Chipman, Russell A., 1A \\ Choi, Daeyoung, $\mathrm{OH}, 0 \mathrm{O}$ \\ Chuang, Ricky W., 16 \\ Citrin, David S., 00 \\ Daami, Anis, $\mathrm{OE}$ \\ Dadlani, Aresh, 19 \\ Dang, Cuong, $1 \mathrm{G}$ \\ da S. Ferreira, Adriano, $1 \mathrm{~N}$ \\ Daubenschüz, Markus, OG \\ Debernardi, Pierluigi, OG \\ Dittrich, P.-G., $\mathrm{OZ}$ \\ Dolgiy, Alexey, 10 \\ Drozdowski, Winicjusz, $1 \mathrm{G}$ \\ El-Adawy, M., IF \\ El-Saeed, Ahmed H., 13 \\ Fantoni, A., 17 \\ Fathpour, Sasan, 15 \\ Gnawali, Rudra, 1Q \\ Goano, Michele, OG \\ Gowda, Uday, OM, 1M \\ Guo, S., OZ \\ Guttmann, Martin, OD \\ Hagras, Esam A. A. A., $1 \mathrm{~J}$ \\ Hameed, Mohamed Farhat $0 ., 13,1 F, 1 H$,

$$
11,1 \mathrm{~J}
$$ \\ Heikal, A. M., $1 \mathrm{~J}$ \\ Helmy, Fatma E., IF \\ Hernández-Figueroa, Hugo E., IN \\ Hettiarachchi, Chathuranga, $1 G$ \\ Hsiao, Yu-Hao, $1 \mathrm{C}$ \\ Hsieh, Chi-Ti, OV, $1 \mathrm{C}$ \\ Huang, Yu-Chun, 16
}

\author{
Hussein, Mohamed, $1 \mathrm{~F}$ \\ Hussein, Rasha A., 11 \\ Hutchins-Delgado, Troy, OI, OX \\ Huyet, Guillaume, OM, $1 \mathrm{M}$ \\ Im, Seongmin, 1D \\ Jean Charles, Estania, OI, OX \\ Kantner, Markus, OU \\ Khalil, Ahmed E., 13 \\ Khinevich, Nadia, 10 \\ Kholyavo, Victor, 10 \\ Kim, Donghyun, 1D \\ Kimura, Ryota, 1A \\ Kitagawa, Selichiro, 1A \\ Kneissl, Michael, OD \\ Knežević, Tihomir, OY \\ Kovalev, Anton V., 0O, $1 \mathrm{M}$ \\ Kovanis, Vassilios, $\mathrm{OQ}$ \\ Kumar, Manish, 14 \\ Kuo, Hao-Chung, $1 \mathrm{C}$ \\ Kurbakov, Igor L., 05 \\ Lai, Ming-Sheng, $1 \mathrm{C}$ \\ Lee, Hosuk, OI, OX \\ Lee, Yao-Jen, 16 \\ Lin, Chih-Hsien, OV, $1 \mathrm{C}$ \\ Liv, Fengqi, ON \\ Locquet, Alexandre, $\mathrm{OH}, 0 \mathrm{O}$ \\ Louro, P., 17 \\ Lozovik, Yurii E., 05 \\ Magana Contreras, Diana, OI, OX \\ Malheiros-Silveira, Gilliard N., IN \\ Malinowski, Marcin, 15 \\ Michalzik, Rainer, OG \\ Nanver, Lis K., OY \\ Nasr, Abed M., $1 \mathrm{H}$ \\ Nazib, Sami Adnan, Ol, OX \\ Nguyen, Tien Hoa, $1 \mathrm{G}$ \\ Nogan, John, Ol, OX \\ Notni, G., $0 Z$ \\ Obayya, S. S. A., 13, 1F, 1H, 11, 1J \\ Olivier, François, OE \\ Onyejegbu, Elochukwu, 19 \\ Osiński, Marek, Ol, OX \\ Park, Hyo-Hoon, 18, 19 \\ Pimenov, Alexander, OM \\ Polvektova, Anel, 19 \\ Radtke, L., OZ \\ Raghuwanshi, Sanjeev Kumar, 14, 1B \\ Rebrova, Natalia, 1M \\ Redko, Sergey, 10
}


Renteria, Emma J., OI, OX

Römer, Friedhard, OD

Rosenberger, M., $0 Z$

Sakarya, Doğan Uğur, 1K, $1 \mathrm{~L}$

Sakr, Hesham, 11

Seigo, Masafumi, 1A

Shaker, Ahmed, IF

Shih, Min-Hsiung, 1C

Sim, Eunji, 1D

Singh, Yadvendra, 14, 1B

Slepneva, Svetlana, OM

Suligoj, Tomislav, OY

Susilo, Norman, OD

Tibaldi, Alberto, OG

Ukaegbu, Ikechi A., 18, 19

Valagiannopoulos, Constantinos, $\mathrm{OQ}$

Vieira, M. A., 17

Vieira, M., 17

Vieira, P., 17

Viktorov, Evgeny A., OH, OM, 0O, $1 \mathrm{M}$

Vladimirov, Andrei G., OM, $1 \mathrm{M}$

Voronova, Nina S., 05

Wang, Hanquan, $\mathrm{ON}$

Wang, Hong, $1 G$

Watad, Ibrahim, OT

Wernicke, Tim, OD

Wishon, Michael J., $\mathrm{OH}, 0 \mathrm{O}$

Withers, Nathan J., OI, OX

Witkowski, Marcin E., IG

Witzigmann, Bernd, OD

Xie, Aozhen, $1 \mathrm{G}$

Yu, Cheng-Li, IC

Zavatski, Siargei, 10

Zhang, C., $\mathrm{OZ}$

Zhang, Jinchuan, ON

Zhigulin, Dmitri, 10

Zhou, Debao, ON 


\section{Conference Committee}

Symposium Chairs

Connie J. Chang-Hasnain, University of California, Berkeley (United States)

Graham T. Reed, Optoelectronics Research Centre, University of Southampton (United Kingdom)

Symposium Co-chairs

Sailing He, KTH Royal Institute of Technology (Sweden) and Zhejiang University (China)

Yasuhiro Koike, Keio University (Japan)

Program Track Chairs

James G. Grote, Photonics Consultant (United States)

Shibin Jiang, AdValue Photonics, Inc. (United States)

Conference Chairs

Bernd Witzigmann, Universität Kassel (Germany)

Marek Osiński, The University of New Mexico (United States)

Yasuhiko Arakawa, The University of Tokyo (Japan)

Conference Program Committee

Hiroshi Amano, Nagoya University (Japan)

Toshihiko Baba, Yokohama National University (Japan)

Jing Bai, University of Minnesota, Duluth (United States)

Enrico Bellotti, Boston University (United States)

Guillermo Carpintero del Barrio, Universdad Carlos III de Madrid (Spain)

Weng W. Chow, Sandia National Laboratories (United States)

Alexandre Freundlich, University of Houston (United States)

Frédéric Grillot, Télécom ParisTech (France)

Ortwin Hess, Imperial College London (United Kingdom)

Stephan W. Koch, Philipps-Universität Marburg (Germany)

Cun-Zheng Ning, Arizona State University (United States)

Joachim Piprek, NUSOD Institute LLC (United States)

Marc Sciamanna, CentraleSupélec (France)

Ikuo Suemune, Hokkaido University (Japan)

Kaikai Xu, State Key Laboratory of Electronic Thin Films \& Integrated Devices (China) 


\section{Session Chairs}

1 Nano-Emitters and Non-Classical Light

Bernd Witzigmann, Universität Kassel (Germany)

2 Quantum Dot Lasers

Yasuhiko Arakawa, The University of Tokyo (Japan)

3 III-Nitride Lasers and LEDs

Andrea Fiore, Technische Universiteit Eindhoven (Netherlands)

4 Lasers and LEDs I

Johann Peter Reithmaier, Universität Kassel (Germany)

5 Laser Dynamics

Marek Osiński, The University of New Mexico (United States)

6 Electromagnetics and Plasmonics

Alberto Tibaldi, Politecnico di Torino (Italy)

7 Lasers and LEDs II

Bernd Witzigmann, Universität Kassel (Germany)

8 Photodetection

Sahar Sharifzadeh, Boston University (United States)

9 Sensors

Markus Kantner, Weierstrass-Institut für Angewandte Analysis und Stochastik (Germany)

10 Optical Systems Simulation

Jing Bai, University of Minnesota Duluth (United States) 